\title{
Current-temperature measurements of a SBD evaporated onto inductively coupled plasma cleaned germanium
}

\author{
S.M.M. Coelho *, F.D. Auret, G. Myburg, P.J. Janse van Rensburg, W.E. Meyer \\ Physics Department, University of Pretoria, Pretoria 0002, South Africa
}

\section{A R T I C L E I N F O}

PACS:

71.20.Mq

73.40.Ei

61.82.Fk

$79.40 .+\mathrm{z}$

$73.30 .+\mathrm{y}$

Keywords:

Germanium

Schottky barrier diode

ICP

DLTS

Defects

Dry etch

\begin{abstract}
A B S T R A C T
Inductively coupled plasma (ICP) etching has been used primarily on compound semiconductors. There are however compelling reasons to study the effects of ICP etching on Ge. Pd Schottky barrier diodes (SBDs) were resistively evaporated onto Ge (111) that was ICP etched at a rate of $60 \AA$ per minute for three or ten minute intervals. Although plasma cleaning is known to introduce defects that were observed with DLTS, the diodes exhibited excellent current-voltage characteristics when cooled down to $80 \mathrm{~K}$. Current-temperature (IT) scans that were recorded from $20 \mathrm{~K}$ up to $300 \mathrm{~K}$ after cooling under reverse bias showed no effect of recombination/generation (RG). On the other hand, IT scans that were recorded after cooling under zero or forward bias clearly exhibited RG effects in the $100-240 \mathrm{~K}$ temperature range. This effect was found to be completely reversible. In addition, ICP etching leads to superior devices when compared to devices manufactured by RF sputter deposition.
\end{abstract}

(C) 2009 Elsevier B.V. All rights reserved.

\section{Introduction}

In the manufacture of semiconductor devices, metal contacts have always played a pivotal role, especially in MOSFET and CMOS devices. Understanding the effect of different metallization techniques on the electrical properties of a semiconductor is of critical importance as we advance into the age of ultrafast devices. Germanium is now receiving renewed attention as its high mobility carriers, both holes and electrons, make it an ideal material for the next-generation fast switching devices. There are also several important niche applications for germanium such as high-resolution gamma-ray detectors, far IR detectors and low temperature thermistors (see [1] and references therein).

Since the 1970s, the need for higher circuit density has resulted in dry etching processes becoming popular. Their advantage over the cost effective wet etches is that dry etches produce higher resolution pattern transfer. Of the many dry etch processes available today, ICP etching even has advantages over electron cyclotron resonance (ECR) etching as it has no DC magnetic field requirement and large wafers can be accommodated [2]. An ICP system operating under optimal conditions can produce ion currents that are uniform to $\pm 1.5 \%$ over a large wafer [3] and this translates into a uniform etch rate across the wafer. It has

\footnotetext{
* Corresponding author. Tel.: +27 12 4205135; fax: +27123625288.

E-mail address: sergio@up.ac.za (S.M.M. Coelho).
}

further been shown that ICP etching produces lower ion damage than traditional plasma etches when applied to compound semiconductors [4-6]. ICP and ECR etches were also found to change the electrical properties of diodes fabricated on compound semiconductors less than conventional reactive ion discharges as RF-induced DC self-biases are much lower in ICP and ECR systems [5].

Device properties will be further influenced by the defects introduced during metallization. High melting point metals cannot be applied onto the semiconductor surface using benign techniques like resistive evaporation, making it necessary to quantify the defects introduced by more energetic deposition processes like electron beam deposition (EBD) and sputter deposition (SD). Defects introduced, unintentionally or intentionally, near the semiconductor-metal interface will alter the barrier heights of contacts influencing device performance $[7,8]$.

\section{Experimental procedures}

Bulk-grown (111) n-type $\mathrm{Ge}$, doped with $\mathrm{Sb}$ to a level of $2.5 \times 10^{15} \mathrm{~cm}^{-3}$ was degreased in successive, five minute, trichloroethylene, acetone and methanol ultrasonic baths. After a de-ionized water rinse, samples were etched in a mixture of $\mathrm{H}_{2} \mathrm{O}_{2}$ (30\%) : $\mathrm{H}_{2} \mathrm{O}(1: 5)$ for one minute, rinsed with de-ionized water and dried with nitrogen gas, then inserted into a vacuum chamber where AuSb $(0.6 \% \mathrm{Sb})$ was deposited on the wafer back surface by 
Table 1

Reverse bias current of Pd Schottky diodes manufactured by different deposition methods.

\begin{tabular}{|c|c|c|c|c|}
\hline Deposition & Cleaning procedure & Current $(\mathrm{A})$ at $-1 \mathrm{~V}$ bias $(100 \mathrm{~K})$ & Current (A) at $-1 \mathrm{~V}$ bias $(300 \mathrm{~K})$ & Ideality factor/barrier height $(\mathrm{eV})$ \\
\hline $\mathrm{RE}$ & Degrease, $\mathrm{H}_{2} \mathrm{O}_{2}$ etch & Below $10^{-14}$ & $2.3 \times 10^{-6}$ & $1.06 / 0.55$ \\
\hline $\mathrm{RE}$ & Degrease, ICP & $\sim 5 \times 10^{-14}$ & $2 \times 10^{-5}$ & $1.15 / 0.53$ \\
\hline $\mathrm{EB}$ & Degrease only & $6 \times 10^{-8}$ & $3 \times 10^{-6}$ & $1.50 / 0.61$ \\
\hline $\mathrm{EB}$ & Degrease, $\mathrm{H}_{2} \mathrm{O}_{2}$ etch & Below $10^{-14}$ & $3.9 \times 10^{-5}$ & $1.30 / 0.52$ \\
\hline $\mathrm{EB}$ & Degrease, 3 min ICP & $\sim 5 \times 10^{-14}$ & $1.5 \times 10^{-5}$ & $1.12 / 0.59$ \\
\hline $\mathrm{EB}$ & Degrease, $10 \mathrm{~min}$ ICP & $\sim 5 \times 10^{-14}$ & $1.5 \times 10^{-5}$ & $1.50 / 0.57$ \\
\hline SD-Au & Degrease, $\mathrm{H}_{2} \mathrm{O}_{2}$ etch & $3 \times 10^{-9}$ & $5.9 \times 10^{-5}$ & $2.05 / 0.44$ \\
\hline
\end{tabular}

resistive evaporation (RE). A ten minute anneal in an $\mathrm{Ar}$ atmosphere at $350^{\circ} \mathrm{C}$ lowered the barrier height resulting in an ohmic contact. Phase two of device fabrication involved cutting the wafer into $3 \mathrm{~mm} \times 5 \mathrm{~mm}$ pieces and the same degreasing procedure as above was applied, followed by mounting of a sample on a holder in a vacuum chamber where plasma cleaning and EBD of the front side of the sample took place through a metal contact mask that has $0.6 \mathrm{~mm}$ diameter holes in it. ICP and EBD were carried out successively without breaking vacuum. Diodes manufactured by RE, a process known not to introduce measurable defects [9], were plasma etched in one vacuum system, while resistive evaporation was performed in a second vacuum system, exposing the Ge samples to the atmosphere while transferring the sample from one system to another. Initial vacuum was better than $10^{-5}$ mbar for all processes regardless of the system used. Ten minute and three minute plasma etches were performed by a Copra DN 200 inductively coupled plasma beam source. Radio Frequency (RF) power of $500 \mathrm{~W}$ forward ( $23 \mathrm{~W}$ reverse) and an argon pressure of $5 \times 10^{-4}$ mbar were the conditions used for all plasma treatments followed by a $100 \mathrm{~nm}$ thick Pd deposition onto the germanium to form the Schottky barrier diodes (SBDs) considered herein. In situ monitoring of the film thickness growth was achieved in the resistive and EB systems via an Inficon crystal growth monitor. Pd was deposited by RE at a rate of $0.3 \mathrm{~nm} / \mathrm{s}$ and a similar EB deposition rate was achieved using a $10 \mathrm{kV}$ electron gun with a beam current of approximately $0.1 \mathrm{~A}$.

Current-voltage (IV) and deep level transient spectroscopy (DLTS) measurements, except for the room temperature dark IV measurements, were performed in vacuum in a closed-cycle helium cryostat.

\section{Results and discussion}

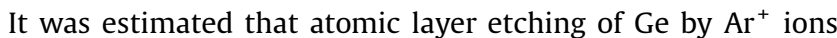
required energy higher than $13 \mathrm{eV}$ [10]. SRIM simulations predicted slightly higher ion energy of $17 \mathrm{eV}$ to dislodge Ge atoms from an amorphous target. ICP etches were carried out with an average $\mathrm{Ar}^{+}$ion energy of $80 \mathrm{eV}$ (as measured by Copra) to minimize the damage caused to the Ge crystal lattice while maintaining a reasonable etch rate of $6 \mathrm{~nm}$ per minute. Eight diodes were deposited on each sample and room temperature. IV measurements were subsequently carried out to ascertain diode quality as well as uniformity across the sample. Ideality factors and the $I V$ barrier height was extracted from a linear fit of the forward bias $\log [I /(1-\exp (-q V / k T))]$ versus $V$ plot [11]. Idealities below 1.1 were considered to be "good" provided that an accurate linear fit to the data was obtained [12]. Reverse bias current at $-1 \mathrm{~V}$ was used as an indication of the diode quality. This current measurement is diode area dependent and for Ge diodes with a diameter of $0.6 \mathrm{~mm}$, values below $10^{-5} \mathrm{~A}$ with at least three orders of magnitude between the $1 \mathrm{~V}$ forward and reverse bias currents are acceptable.

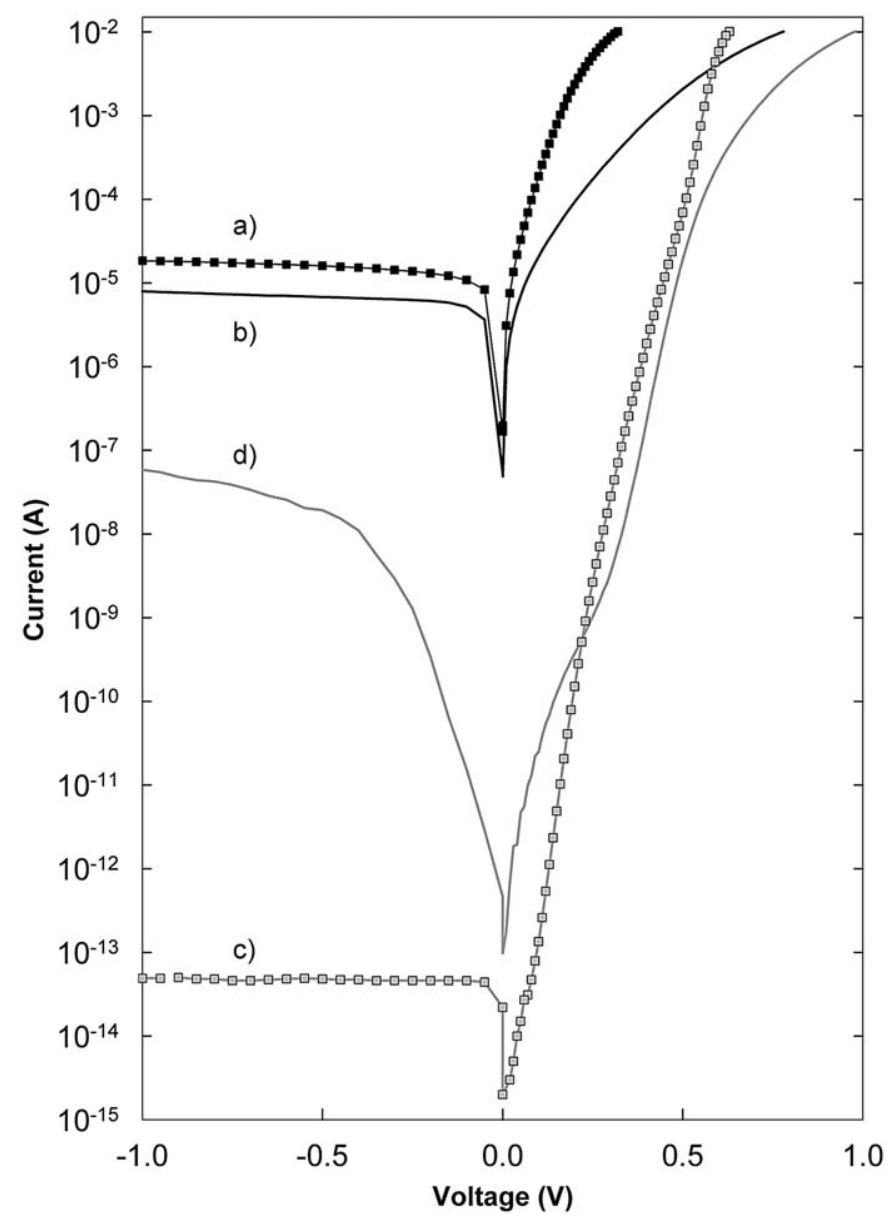

Fig. 1. Current-voltage measurements under forward and reverse bias conditions for Pd Schottky diodes, recorded at 300 and $100 \mathrm{~K}$, respectively: (a) 10 min plasma etched EB deposited diode measured at $300 \mathrm{~K}$, (b) unetched EB deposited diode measured at $300 \mathrm{~K}$, (c) diode (a) measured at $100 \mathrm{~K}$ and (d) diode (b) measured at $100 \mathrm{~K}$.

ICP etching and EBD are capable of introducing defects at and below the semiconductor surface as energetic particles are accelerated in both processes and these interacted with the Ge samples. Thus, while it is not surprising that the RE deposited Schottky barrier diode had an ideality closest to one and a low $1 \mathrm{~V}$ reverse bias current of $2.3 \times 10^{-6} \mathrm{~A}$ as summarized in Table 1 , the diode produced by 3 min ICP etching followed by EBD had a slightly worse ideality of 1.12 with a room temperature $1 \mathrm{~V}$ reverse bias current that was almost one order of magnitude higher. When these two diodes are compared at $100 \mathrm{~K}$ both diodes exhibited $1 \mathrm{~V}$ reverse bias currents that were low enough to be at the lower limit measurable by the HP $4140 \mathrm{~B}$ pA meter. This result was in sharp contrast to the diode produced by EB deposition without ICP that had a $1 \mathrm{~V}$ reverse bias current six orders of 


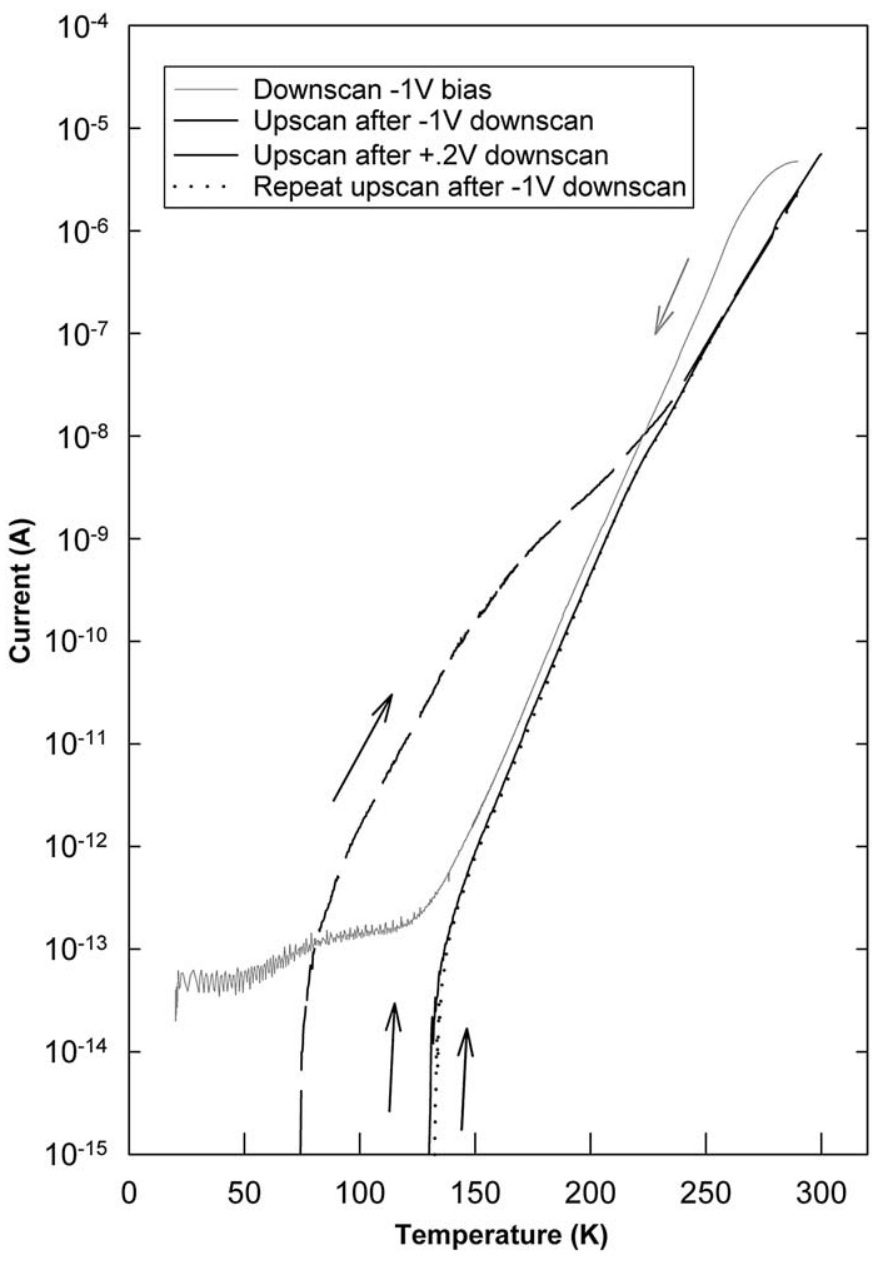

Fig. 2. Cooling under forward and reverse bias and its effect on Currenttemperature measurements of ICP etched Ge.

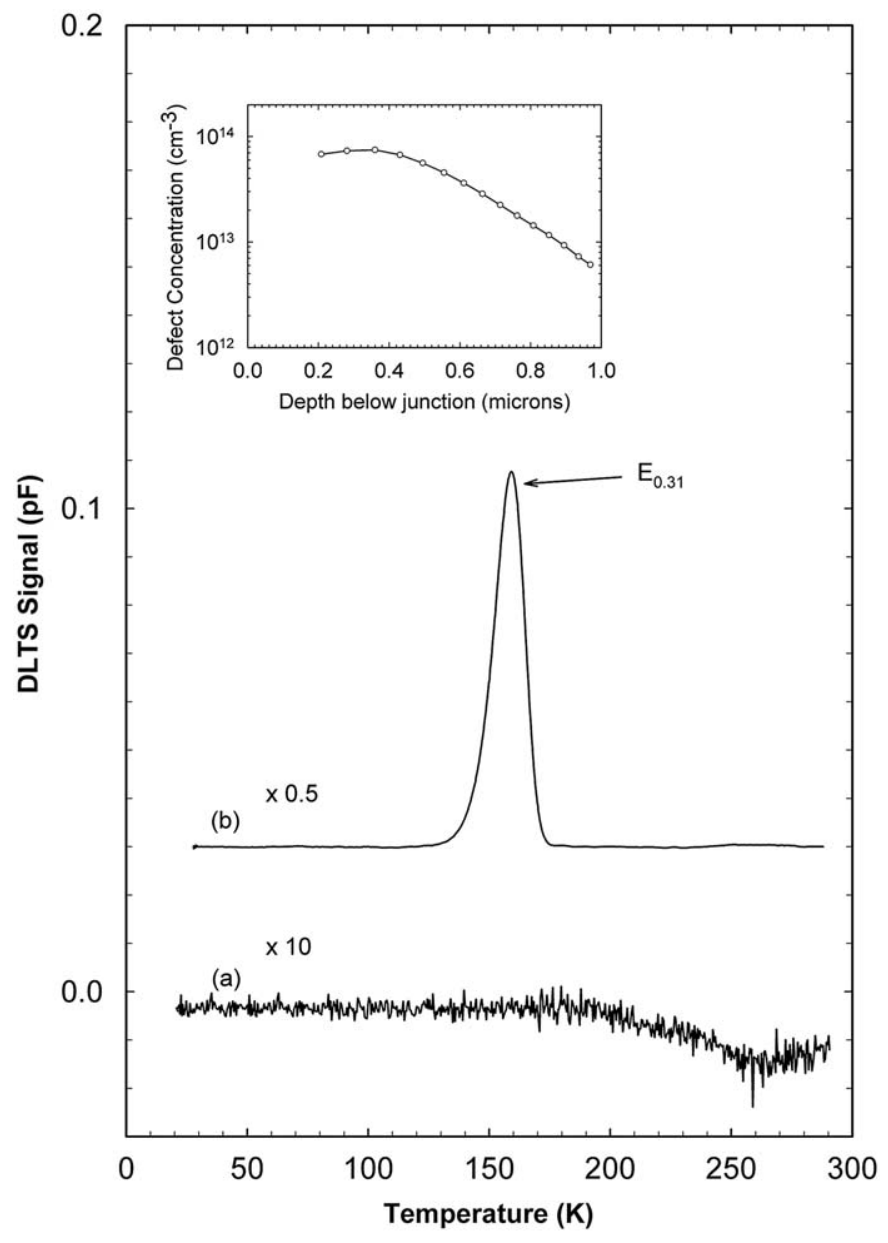

Fig. 3. DLTS electron trap spectra of resistively evaporated Pd SBD's where (a) is the control sample and (b) was ICP cleaned for $10 \mathrm{~min}$. Spectra were recorded using a rate window of $80 \mathrm{~s}^{-1}$ and a quiescent reverse bias of $1 \mathrm{~V}$ with a superimposed $1.2 \mathrm{~V}$ filling pulse, $V_{p}$. Inset: Electron defect $\left(E_{0,31}\right)$ concentration below the junction of the ICP cleaned sample.

cooled from 300 to $20 \mathrm{~K}$ (downscan) is not only linear but also very similar to the plot taken while heating the diode from 20 to $300 \mathrm{~K}$ (upscan) at a scan rate of $5 \mathrm{~K}$ per minute, similar to the downscan rate. The diode was then subjected to another cycle of cooling with a $0.2 \mathrm{~V}$ forward bias applied followed by an upscan at $-1 \mathrm{~V}$ bias. A significant deviation from the previous ideal behaviour was observed and the increase in current from 70 to $240 \mathrm{~K}$ is evidence of recombination/generation effects, since empirically the current, $J$, is proportional to $\exp (-q V / 2 k T)$ if the recombination current dominates and is proportional to $\exp (-q V / k T)$ if the diffusion current dominates [13]. Cooling under forward bias filled the traps which then released electrons as the sample was heated. Cooling the diode again under reverse bias of $1 \mathrm{~V}$ fully restored the device and the upscan that followed was indistinguishable from the initial upscan.

DLTS measurements of this diode confirmed that this defect was indeed an electron trap with energy of $0.31 \mathrm{eV}$ below the conduction band and a distribution of at least $1 \mu \mathrm{m}$ into the Ge (Fig. 3). No hole traps were observed. SRIM predicted $\mathrm{Ar}^{+}$events that were more than three hundred times shallower than the distribution observed and this suggests that this defect may be mobile at room temperature. Defects that have previously been observed by DLTS in EBD Pd SBDs were absent in all ICP treated samples and warrants further investigation. 


\section{Conclusions}

ICP is a suitable cleaning procedure for germanium that introduced defects but had no adverse effect on diode quality. ICP cleaned Ge exhibits excellent low temperature IV characteristics with good recovery properties. SRIM simulations suggest that the ICP introduced defect should not be as deep into the material as observed, unless it is mobile at room temperature. The absence of other measurable EBD-induced defects may indicate that ICP etching traps these defects at the Ge surface and bears further investigation.

\section{Acknowledgement}

The authors gratefully acknowledge the financial support of the South African National Research Foundation.

\section{References}

[1] E.E. Haller, Mater. Sci. Semicond. Process. 9 (2006) 408.

[2] S.J. Pearton, D.P. Norton, Plasma Process. Polym. 2 (2005) 16

[3] R.W. Boswell, A.J. Perry, M. Emami, J. Vac. Sci. Technol. A 7 (6) (1989) 3345.

[4] R.J. Shul, G.B. McClellan, R.D. Briggs, D.J. Rieger, S.J. Pearton, C.R. Abernathy, J.W. Lee, C. Constantine, C. Barratt, J. Vac. Sci. Technol. A 15 (3) (1997) 633.

[5] J.W. Lee, C.R. Abernathy, S.J. Pearton, F. Ren, W.S. Hobson, C. Constantine, C. Barratt, J. Electrochem. Soc. 144 (4) (1997) 1417.

[6] S.J. Pearton, Appl. Surf. Sci. 117/118 (1997) 597.

[7] F.D. Auret, W.E. Meyer, S. Coelho, M. Hayes, Appl. Phys. Lett. 88 (2006) 242110

[8] J.D. Waldrop, Appl. Phys. Lett. 44 (1984) 1002.

[9] F.D. Auret, W.E. Meyer, S. Coelho, M. Hayes, Appl. Phys. Lett. 88 (2006) 242110.

[10] T. Sugiyama, T. Matsuura, J. Murota, Appl. Surf. Sci. 112 (1997) 187.

[11] J.D. Waldrop, Appl. Phys. Lett. 44 (1984) 1002.

[12] E.H. Rhoderick, R.H. Williams, Metal-Semiconductor Contacts, Clarendon Press, Oxford, 1988.

[13] S.M. Sze, K.K. Ng, Physics of Semiconductor Devices, Wiley, New York, 2007. 